\title{
System Classification by Using Discriminant Functions of Time-Frequency Features
}

\author{
Miguel Mendoza Reyes ${ }^{1}$, Juan V. Lorenzo-Ginori ${ }^{1}$, \\ A. Taboada-Crispí ${ }^{1}$, and Yakelin Luna Carvajal ${ }^{2}$ \\ ${ }^{1}$ Center for Studies on Electronics and Information Technologies, Universidad Central \\ "Marta Abreu" de Las Villas, Carretera a Camajuaní, km 5 1⁄2, Santa Clara, VC, Cuba, \\ CP 54830 \\ \{mmendoza, juanl, ataboada\}@uclv.edu.cu \\ ${ }^{2}$ Ministry of Public Health, Santa Clara, VC, Cuba \\ yakelin@uclv.edu.cu
}

\begin{abstract}
Time-frequency representations (TFR) convey relevant information about systems that can not be obtained under stationary conditions. In this paper, a methodology to classify systems using the information obtained from time-frequency representations during transient phenomena is described and tested experimentally. The study includes an assessment of the features to be extracted from the TFR, which are relevant for the desired classification, as well as the construction of the appropriate discriminant functions using them. The methodology is tested by means of a biomedical example related to patient's classification.
\end{abstract}

Keywords: time-frequency distributions, feature extraction.

\section{Introduction}

System classification can be done through the analysis of the signals they produce. When the analysis is to be done in the frequency domain, there are considerations that should be addressed. In the case of a signal whose spectral characteristics vary with time, if there is a need of knowing the spectral content of that signal for every time instant, in order to perform a dynamic spectral analysis, the use of a time-frequency representation (TFR) of that signal is commonly required. This dynamic representation of the spectral behavior can be obtained by processing signals through the time-frequency distributions (TFD).

From the TFR of signals, a set of features can be extracted to characterize them. The selection of the most appropriated features depends mainly on the type of TFD used to process the signal, and the application where they will be employed. For those TFR that allow energetic interpretations, features values serve to estimate the form in which signal energy is locally distributed in the time-frequency $(\mathrm{t}-\mathrm{f})$ plane. For TFR computation, TFD from the Cohen group have been commonly assessed. The TFDs from this group of bilinear distribution have the general form

$$
C_{x}(t, f ; \varphi)=\int_{-\infty}^{\infty} \int_{-\infty}^{\infty} \int_{-\infty}^{j 2 \pi \theta(u-t)} z(u+\tau / 2) z^{*}(u-\tau / 2) \varphi(\theta, \tau) e^{-j 2 \pi f \tau} d \theta d u d \tau,
$$


where $\mathrm{z}(\mathrm{t})$ is the analytic signal and $\varphi(\theta, \tau)$ the kernel function, whose behavior in the $(\theta, \tau)$ plane, or ambiguity domain, defines the general properties of the TFD 1.

A typical TFR contains a large amount of information. To reduce this, features are extracted from particular zones in the t-f plane. The size and location of these zones are mainly defined according to the specific application. After computing the features, the selection of the suitable ones is a mandatory task in the classification procedures.

To determine the feasibility of features for classification purposes, they must be evaluated in order to find their discriminatory possibilities. The combination of these selected features in discriminant functions allows system classification.

All the process should be repeated in order to find the configuration with the greater amount of features with discriminatory possibilities. This process includes the selection of the appropriate TFD used to obtain the TFR, and the sizes and location of the proper zones in TFR from which the features will be extracted.

This paper presents a methodology for system classification by using discriminant functions of statistical parameters of time-frequency features. Section two depicts the methodology proposed and describes the systems under classification. The methods used for the computation of TFR and its features, and the selection of statistical parameters and its combination, are also presented in this section. Section three shows the results of the system classification by following the proposed methodology. Finally, section four encloses the discussion of obtained results and resulting conclusions.

\section{Materials and Methods}

The global procedure followed to find the discriminant functions could be summarized in the following steps:

1. Set initial configuration for TFR computation: TFD type and parameters, window type and parameters.

2. Select zones of TFR to compute features: (depending on application)

3. Extract features from TFR zones.

4. Compute statistical parameters of features in selected zones.

5. Select parameters for analysis of variance (ANOVA): normality and homogeneity test.

6. Select parameters with discriminatory abilities: ANOVA and multiple comparisons.

7. Select the setup for TFR computation according to the number of parameters with discriminatory abilities.

8. Find combinations of parameters to form discriminant functions: LDA

9. Test discriminant functions: Wilk's lambda, Cross-validation.

In the present work, the systems to be classified were patients with different blood pressure (BP) behavior and therefore different influence on their autonomic nervous system (ANS). ANS controls heart rate through its sympathetic and parasympathetic 
branches. High blood pressure (HBP) can affects the performance of the ANS and, as a result, also influences the behavior of the heart rate variability (HRV) signal 234.

Spectro-temporal features of HRV, derived from an appropriate TFR, were used in this example for the classification of patients with different BP behavior. Features selection was done according to the proposed methodology.

\subsection{Patients}

Patients under study belonged to one of three categories: hyperreactive (HR) that comprised individuals whose levels of cardiovascular response to the left-arm isometric exercise (LAIE) were greater than the limits set to the normotensive (NT) group, but lower than those set for the hypertensive (HT). HR group has been considered prone to develop HBP 5.

The LAIE consists of three stages. During the first one, lasting two minutes, the rest stage, the patient sits relaxed with his hands on his legs. Then the effort stage begins, the patient extends his left arm horizontally with respect to the floor, while holding a 500 gram weight during two minutes. Finally, the patient releases the weight and put his left arm back to the rest position, keeping it that way for the two minutes that last this final recovery stage. The whole procedure lasts for six minutes.

The group of patients comprised 27 individuals, all male. Subjects with health conditions that are known, or suspected, to cause autonomic disorders, such as diabetes, epilepsy and asthma, were excluded.

Patients were selected according to confirmed diagnosis reached by a team of physicians after various studies and laboratory tests. The characteristics of the groups evaluated are shown in table 1.

Table 1. Groups of patients

\begin{tabular}{lccl}
\hline Group & & Number of patients & Age $(\mathrm{m} \pm$ std), years \\
\hline Normotensive & (NT) & 11 & $31.18 \pm 5$ \\
Hypertensive & (HT) & 10 & $41.20 \pm 7$ \\
Hyperreactive & (HR) & 6 & $36.85 \pm 12$ \\
\hline
\end{tabular}

m: mean value, std: standard deviation

\subsection{TFD Configuration}

Spectral analysis of HRV is the recommended method when the study of the effect of one of the systems that modulates this signal through the ANS is needed 3. To highlight the influence of a particular system on the ANS, HRV is commonly obtained from electrocardiographic (ECG) records acquired during the execution of exercises 26 , as in this case. Due to the non-stationary characteristics of such records, the use of time-frequency analysis (TFA) is the most suitable approach for HRV dynamic spectral analysis 1 .

In HRV studies, Wigner-Ville Distribution (WVD) and its smoothed versions have been commonly used 7-10. Choi-Williams distribution (CWD), a representative case 
of the reduced-interference distributions (RID) group, have also been addressed 11. In this study TFR were obtained by using the Choi-Williams distribution,

$$
C W D_{x}(t, f)=\sqrt{\frac{2}{2}} \int_{-\infty}^{\infty} \int_{-\infty}^{\infty} \frac{\sigma}{|\tau|} e^{-2 \tau^{2}(u-t)^{2} / \tau^{2}} z(u+\tau / 2) z^{*}(u-\tau / 2) \varphi(v, \tau) e^{-j 2 \pi f \tau} d u d \tau
$$

and the smoothed-pseudo-Wigner-Ville distribution,

$$
S P W V D_{x}(t, f)=\int_{-\infty}^{\infty} h(\tau) \int_{-\infty}^{\infty} g(u-t) z(u+\tau / 2) z^{*}(u-\tau / 2) e^{-j 2 \pi f \tau} d u d \tau
$$

where $h(\tau)$ is the frequency smoothing window or data window, $g(u)$ is the time smoothing window and $z(t)$ is the analytic version of time signal $x(t) 7$.

In (2), the parametrization function, or distribution kernel, $\varphi(v, \tau)$, has the form

$$
\varphi_{(v, \tau)}=e^{-\frac{(\pi v \tau)^{2}}{2 \sigma^{2}}}
$$

In this study, the value $\sigma=0.1$ was used to favor interference reduction 12 .

\subsection{Feature Extraction}

After computing the TFR of HRV, there were extracted instantaneous estimates of frequency, power and bandwidth from the LF band, $0.04 \mathrm{~Hz}$ to $0.15 \mathrm{~Hz}$, and from $\mathrm{HF}$ band, $0.15 \mathrm{~Hz}$ to $0.4 \mathrm{~Hz}$, as defined in 3 These features have been linked to the ANS in previous studies 236 . Their values were computed from the moments and marginals of TFRs 17 . A list of the TFR features evaluated in this study is presented in table 2 .

Table 2. TFR features evaluated in this study

\begin{tabular}{cl}
\hline Feature & \multicolumn{1}{c}{ Meaning } \\
\hline IF & Instantaneous frequency \\
IB & Instantaneous bandwidth \\
IP & Instantaneous power \\
PS & Power spectral density \\
E & Local energy \\
\hline
\end{tabular}

The feature vectors, containing the values computed for the whole record, were divided according to the three exercise stages. Stages 2, effort, and 3, recovery, were subsequently divided in sections where statistical parameters were computed.

An appropriate configuration was elected after considering several alternatives. These involved different analysis windows, TFDs, and number of sections in which 
the stages of effort and recovery were divided. A preliminary selection of the configuration was made heuristically.

The statistical parameters evaluated were: mean value, $\mathrm{M}$, trimmed mean, TM, and standard deviation, SD. The values corresponding to the effort and recovery stages were normalized with respect to those corresponding to the rest stage, to reduce interindividual variations.

The names of the parameters were formed by appending the numbers of the corresponding stage and section, in that order, to the name of the feature. For example, L_H31SD represents the standard deviation of the values of the instantaneous LF to HF bands power ratio, computed for the first section of the recovery stage. MATLAB toolboxes were used for TFR computation and feature extraction 13 .

\subsection{Parameter Selection}

For each evaluated configuration the parameters with higher probability to discriminate patients, according to the group to which they belonged, were searched. One-way ANOVA was used to determine if the parameter under test was significantly different in the groups evaluated, $\mathrm{p}<0.05$. Previously to ANOVA analysis, these parameters were analyzed to ensure distribution normality and homogeneity of variances. Anderson-Darling test and Levene's test were used, respectively, for these purposes 14.

Parameters selected, according to ANOVA results, were further evaluated by using the Tukey-Kramer's multiple comparisons test, to investigate their possibilities for the discrimination of at least one group from the others. Statistical processing was performed with SPSS 15.

\subsection{Discriminant Analysis}

Linear discriminant analysis (LDA) was performed in order to find functions $f_{i}\left(p_{k}\right)$ of the $N$ previously selected parameters $p_{k}$, suitable for the classification of patients. These functions have the form,

$$
f_{i}\left(p_{k}\right)=\sum_{k=0}^{N-1} a_{i k} p_{k},
$$

where

$i=1$..G- 1 , is the function number,

$\mathrm{G}=3$, is the number of groups,

$a_{i k}$ : are the function coefficients.

The Mahalanobis distance in a stepwise analysis was used to obtain the functions. A significance level $p<0.05$ was selected for the inclusion of parameters. Functions were tested using leave-one-out cross-validation, a process in which every case is classified by using discriminant functions obtained from the rest of them. 


\section{Results}

\subsection{TFD Configuration Selection}

Among the different configurations assessed to obtain the TFR, the one that used the CWD with Gaussian windows and six sections per stage had the greater amount of parameters with discriminatory possibilities, $\mathrm{p}<0.05$. The list of parameters and the corresponding $\mathrm{p}$ vales are shown in table 3.

According to multiple comparisons tests, none of the listed parameters was able to differentiate every group from the rest. For example, parameter L_H31SD, the standard deviation of the power ratio in the first section of recovery stage, was significant different for the group HT, but it could not discriminate the groups NT and HR. Figure 1 shows the time progression of this parameter during the effort ant recovery stage.

Table 3. Parameters selected according to established criteria

\begin{tabular}{lc}
\hline Parameter & $\mathrm{p}$ \\
\hline L_H31SD & 0.002 \\
IPL31M & 0.006 \\
IPL31MT & 0.009 \\
IFH31DSD & 0.012 \\
L_H32DSD & 0.015 \\
IPL31SD & 0.017 \\
IFL34DMT & 0.034 \\
IFH22DMT & 0.036 \\
IFL26SD & 0.038 \\
IFL34DM & 0.039 \\
IFH22DM & 0.040 \\
IFL35DM & 0.047 \\
\hline
\end{tabular}

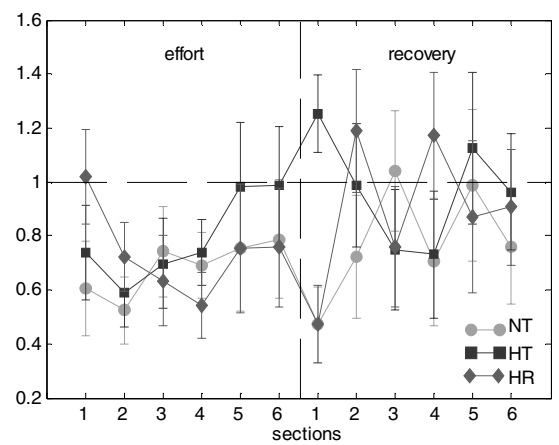

Fig. 1. Time progression of the parameter L_HSD

\subsection{Discriminant Functions}

Two discriminant functions were obtained from the set of selected parameters. The coefficients are shown in table 4. 
Table 4. Coefficients of discriminant functions

\begin{tabular}{clll}
\hline$k$ & \multicolumn{1}{c}{$p_{k}$} & \multicolumn{2}{c}{ Coefficients } \\
& & \multicolumn{1}{c}{$a_{1 k}$} & \multicolumn{1}{c}{$a_{2 k}$} \\
\hline 0 & (constant) & 2,075 & $-0,996$ \\
1 & L_H31SD & 0,114 & 0,270 \\
2 & IFH31DSD & 162,845 & 37,339 \\
3 & IFL34DMT & 157,605 & $-23,029$ \\
4 & IFH22DMT & $-917,906$ & 756,490 \\
5 & IFL26SD & $-290,709$ & 14,561 \\
6 & IFH22DM & 832,625 & $-763,422$ \\
7 & IFL35DM & 75,769 & $-64,371$ \\
\hline
\end{tabular}

The feasibility of the use of discriminant functions for the classification of different cases was tested by determining Wilks' Lambda and significance value, Sig, table 5.

Table 5. Discriminant functions evaluation

\begin{tabular}{ccrcc}
\hline Function & Wilks' Lambda & Chi-square & df & Sig. \\
\hline 1 through 2 & 0.051 & 62.351 & 14 & 0.000 \\
2 & 0.407 & 18.861 & 6 & 0.004 \\
\hline
\end{tabular}

The low value of Wilks' lambda, below 0.1 , and the null value of significance, confirmed that the mean values of the functions were different for the groups, and proved the sufficiency of their application as discriminant functions. Figure 2 shows the scatter plot of the cases, classified by using the discriminant functions.

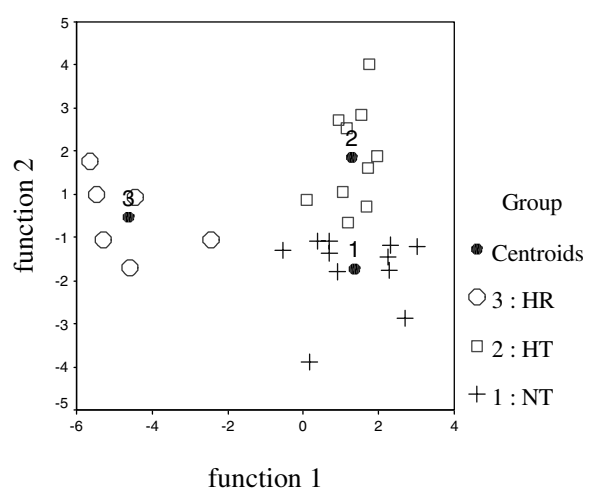

Fig. 2. Scatter plot for all groups

To get a more realistic evaluation of discriminant functions, leave-one-out crossvalidation was performed; table 6 shows the results of this evaluation. 
Table 6. Classification results with original cases and cross-validated evaluation

\begin{tabular}{ccccccc}
\hline & & \multicolumn{5}{c}{ Predicted Group } \\
& & group & NT & HT & HR & Total \\
\hline \multirow{3}{*}{ Original } & \multirow{2}{*}{ cases } & NT & 11 & 0 & 0 & 11 \\
Data & & HT & 1 & 9 & 0 & 10 \\
& & HR & 0 & 0 & 6 & 6 \\
\cline { 3 - 7 } & \multirow{2}{*}{$\%$} & NT & 100.0 & 0.0 & 0.0 & 100.0 \\
& & HT & 10.0 & 90.0 & 0.0 & 100.0 \\
& & HR & 0.0 & 0.0 & 100.0 & 100.0 \\
\hline \multirow{3}{*}{ Cross- } & \multirow{2}{*}{ cases } & NT & 10 & 1 & 0 & 11 \\
Validated & & HT & 3 & 7 & 0 & 10 \\
& & HR & 0 & 0 & 6 & 6 \\
\cline { 3 - 7 } & \multirow{2}{*}{$\%$} & NT & 90.9 & 9.1 & 0.0 & 100.0 \\
& & HT & 30.0 & 70.0 & 0.0 & 100.0 \\
& & HR & 0.0 & 0.0 & 100.0 & 100.0 \\
\hline
\end{tabular}

The difference between classification results obtained with original data from those of cross-validation is satisfactory according to the sample size. This suggests that the number of predictors accounted in the discriminant functions is acceptable.

\section{Discussion and Conclusions}

Due to the great amount of parameters that can be extracted from a TFR, the search of the appropriate features for classification purposes should be done through a combination of techniques.

The proposed methodology contributed to control the amount and quality of parameters with discriminatory abilities. The appropriate selection of feature parameters benefits dimensionality reduction, which is an almost obligatory process in the case of TFR analysis, due to the high dimensionality of the data to be processed.

The discriminant functions, obtained by following the proposed approach, gathered the most appropriate statistical parameters of spectro-temporal features of the signal used to represent the systems under study.

Cross-validation should be used to confirm classification results obtained with the discriminant functions. This procedure generates more realistic results, and is particularly useful if the number of available cases is small.

\section{Acknowledgments}

The authors wish to acknowledge M. D. Alina Pérez-de-Armas for her invaluable help in the completion of this work. We also thank Dr. Ricardo Grau-Abalo and Dr. Gladys Casas-Cardoso for their thorough review of the statistical issues. This research was partially supported by the Canadian International Development Agency Project Tier II-394-TT02-00 and by the Flemish VLIR-UOS Programme for Institutional University Co-operation (IUC). 


\section{References}

1. L. Cohen, "Time Frequency Distribution - A Review", in Proceedings of IEEE, vol. 77, July, 1989, pp 941-981.

2. D. Luccini et al, "Impairment in Cardiac Autonomic Regulation Preceding Arterial Hypertension in Humans. Insights from Spectral Analysis of Beat-by-Beat Cardiovascular Variability," Circulation, November, 2002, pp. 2673-2679.

3. Task Force of European Society of Cardiology and the North American Society of Pacing and Electrophysiology, "Heart Rate Variability. Standards of Measurement, Physiological Interpretation and Clinical Use", European Heart Journal, Vol. 17, pp. 354-381, 1996.

4. L. R. Davrath, Y. Goren, I. Pinhas, E. Toledo and S. Akselrod, "Early autonomic malfunction in normotensive individuals with a genetic predisposition to essential hypertension," Am J Physiol Heart Circ Physiol, vol. 285, pp. H1697-H1704, 2003. Available: http://ajpheart.physiology.org/cgi/content/short/285/4/H1697.

5. M. Benet, J. Apollinaire, J. Torres and S. Peraza, "Reactividad cardiovascular y factores de riesgos cardiovasculares en individuos normotensos menores de 40 años," Revista Española de Salud Pública, vol. 77, no. 1, pp. 143-150, 2003.

6. H. G. Steenis, W. L. J. Martens and J. H. M. Tulen, "Time-Frequency Parameters of Heart rate Variability," IEEE Engineering in Medicine and Biology, vol. 21, no. 4, July / August 2002, pp. 46-58.

7. M. Houle and G. Billman, "Low-frequency component of the heart rate variability spectrum: a poor marker of sympathetic activity," Am J Physiol Heart Circ Physiol, vol. 276, no. 1, pp. H215-H223, January 1999.

8. M. Ebden, L. Tarassenko, S. Payne, A. Darowski A. and J. Price, "Time-frequency analysis of the ECG in the diagnosis of vasovagal syndrome in older people," in Proceedings of the 26th Annual International Conference of the IEEE EMBS, San Francisco, CA, USA, September 1-5, 2004, pp. 290-293.

9. K. Yoshiuchi et al, "Use of time-frequency analysis to investigate temporal patterns of cardiac autonomic response during head-up tilt in chronic fatigue syndrome," Autonomic Neuroscience: Basic and Clinical, vol. 113, pp. 55-62, 2004.

10. S. Jasson et al, "Instant Power Spectrum Analysis of Heart Rate Variability During Orthostatic Tilt Using a Time-/Frequency-Domain Method," Circulation, vol. 96, pp. 3521-3526, July, 1997.

11. F. Clariá, M. Vallverdú, R. Baranowski , L. Chonowska, P. Martínez and P. Caminal , "Time-Frequency Representation of the HRV: A Tool to Characterize Sudden Cardiac Death in Hypertrophy Cardiomyopatthy Patients," in Proceedings of the 22nd Annual EMBS International Conference, Chicago, IL, July 23-28, 2000, pp. 71-73.

12. W. Williams and J. Jeong, "Reduced Interference Time-Frequency Distributions," in Time-Frequency Signal Analysis, B. Boashash, Ed. Longman Cheshire, 1992, pp. 74-97.

13. F. Auger, P. Flandrin, P. Goncalves and O. Lemoine, Time Frequency Toolbox for use with MATLAB, Available: http://www-isis.enst.fr/Applications/tftb/iutsn.univ-nantes.fr/ auger/tftbftp.html.

14. J. L. Romeu, "Anderson-Darling: A Goodness of Fit Test for Small Simples Assumptions," RAC START, vol. 10, no. 5. Available: http://rac.alionscience. com/pdf/A_DTest.pdf.

15. SPSS for Windows, Release 9.01, 1999. 